\title{
Fluoroquinolone und Tendinopathien: Ein Klasseneffekt?
}

B. Schnyder, P. Caduff

\section{Einleitung}

Fluoroquinolone gelten als relativ gut verträgliche Antiinfektiva, doch bestehen zwischen verschiedenen Präparaten dieser Substanzgruppe erhebliche Unterschiede nicht nur betreffend antibakterielle Wirkung, sondern auch Toxizität [1-3]. Während einzelne Präparate sich in langjähriger Praxis als gut verträglich und sicher erwiesen, traten bei anderen zum Teil nicht akzeptable unerwünschte Arzneimittelwirkungen auf (Tab. 2). Bei der Wahl einer Quinolontherapie sollte deshalb das präparatespezifische Nebenwirkungsprofil berücksichtigt werden (Tab. 3). Inwieweit Unterschiede zwischen einzelnen Präparaten auch für Tendinopathien bestehen, wird in der Literatur bis anhin kaum behandelt.

\section{Pathophysiologische und präklinische Aspekte}

Der Pathomechanismus der Quinolon-assoziierten Tendinopathien ist nicht genau bekannt. In der Literatur diskutiert werden hauptsächlich direkt toxische Effekte, ischämische Prozesse [4] und/oder die Chelatbildung mit Magnesiumund anderen Ionen, was in Gewebsstrukturen mit schlechter Durchblutung wie Sehnen oder Knorpel zu einem lokalen Mangel mit den entsprechenden Folgeschäden führen soll $[5,6]$. Fluoroquinolone zeigen in präklinischen Studien eine Toxizität auf den unreifen Gelenksknorpel verbunden mit Wachstumsstörungen, eine Hemmung der Kollagensynthese und antiproliferative respektive zytotoxische Effekte auf Tendinozyten [7]. In Tierversuchen waren die Auswirkungen auf Bindegewebsstrukturen je nach Präparaten unterschiedlich. Die Tendinotoxizität bei Ratten war Fleroxacin = Pefloxacin $>$ Ofloxacin $=$ Levofloxacin $>$ Norfloxacin $=$ Ciprofloxacin [8]. Die Resultate sind aber wegen erheblicher artspezifischer Unterschiede von Stoffwechsel und Durchblutung nicht einfach von der Ratte auf den Menschen übertragbar.

\section{Klinische Aspekte}

Tendinitiden und Sehnenrupturen unter Fluoroquinolontherapie wurden 1983 [9] bzw. 1991 [10] erstmals beschrieben. Für das Auftreten einer Quinolon-assoziierten Tendinopathie wurden mehrere zusätzliche Risikofaktoren identifiziert (Tab. 4).

In einer Zusammenstellung von 100 Fällen mit Quinolon-assoziierter Achilles-Tendinitis [11] schwankte das zeitliche Intervall zwischen Therapiebeginn und dem Auftreten von Symptomen von 1 bis 90 Tage und betrug im Mittel 13 Tage. Betroffen waren in erster Linie die Achillessehnen. In nahezu der Hälfte aller Fälle trat die Entzündung beidseitig auf. In 31\% kam es zu einer Ruptur. Beschwerden treten meist akut auf und sind häufig durch Schmerzen bei Bewegung und Palpation charakterisiert. Starke und stechende Schmerzen weisen auf eine drohende Ruptur hin. Mögliche Zusatzuntersuchungen sind Sonographie und MRI [12].

\section{Epidemiologie}

Die in der Literatur beschriebene Inzidenz von Achillessehnenrupturen in der Allgemeinbevölkerung schwankt je nach Zeitperiode und Studie beträchtlich von 0,47 bis $3,7 / 10000$ [13, 14]. Zwei Kohortenstudien untersuchten die Inzidenz von Tendinopathien in einer breiten Patientenpopulation unter antibiotischer Behandlung $[15,16]$. Beide Studien zeigten unter Fluoroquinolonen eine tendenziell höhere Inzidenz, wobei bei den Quinolonen erhebliche präparatespezifische Unterschiede bestanden und in beiden Studien die Inzidenz von den untersuchten Präparaten bei Ofloxacin am höchsten war (Tab. 5). In einer Studie [15] wurde das relative Risiko im Vergleich zu den Referenztherapien (Amoxicillin, Trimethoprim, Cotrimoxazol oder Nitrofurantoin) berechnet und betrug für die AchillesTendinitis unter Ofloxacin 10,1 (95\% Konfidenzintervall 2,2-46), unter Ciprofloxacin, Norfloxacin und bei Poolen der drei Quinolone waren
E-Mail:

benno.schnyder@swissmedic.ch
Korrespondenz: Swissmedic Erlachstrasse 8 H-3000 Bern 9 
Tabelle 1

In der Schweiz derzeit vermarktete Quinolonpräparate.

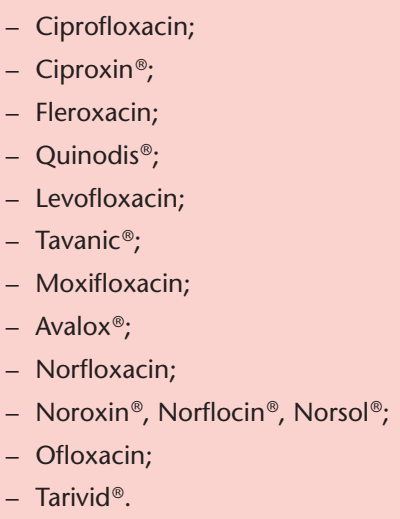

Tabelle 2

Quinolonpräparate mit nicht akzeptablen Nebenwirkungen.

\begin{tabular}{|c|c|c|}
\hline Temafloxacin & $\begin{array}{l}1992 \text { vom US-Markt zurückgezogen, } \\
\text { wurde in CH nie vermarktet }\end{array}$ & $\begin{array}{l}\text { Komplexe Symptomatik } \\
\text { mit Hypoglykämien, } \\
\text { hämolytischen Anämien u.a. }\end{array}$ \\
\hline Sparfloxacin & seit 1997 in $\mathrm{CH}$ nicht mehr vermarktet & Kardio- und Phototoxizität \\
\hline Trovafloxacin & 1999 Abregistrierung & Hepatotoxizität \\
\hline Grepafloxacin & 1999 vom Markt zurückgezogen & $\begin{array}{l}\text { Kardiale und gastrointestinale } \\
\text { Toxizität }\end{array}$ \\
\hline Clinafloxacin & Entwicklung gestoppt & Phototoxizität \\
\hline
\end{tabular}

Tabelle 3

Typische Nebenwirkungen von in der Schweiz gebräuchlichen Quinolonpräparaten [1-3]

\begin{tabular}{|c|c|}
\hline Gastrointestinale Unverträglichkeit & alle \\
\hline Hautreaktionen (Phototoxizität, Exantheme) & alle, speziell Fleroxacin \\
\hline $\begin{array}{l}\text { ZNS-Reaktionen (Kopfschmerzen, } \\
\text { Benommenheit, Schwindel, Schlaflosigkeit, } \\
\text { psychotische Reaktionen, Krampfanfälle) }\end{array}$ & alle, speziell Fleroxacin und Ofloxacin \\
\hline Lebertoxizität & alle \\
\hline Kardiotoxizität mit QTc-Verlängerung & $\begin{array}{l}\text { gezeigt bei Moxifloxacin } \\
\text { und neuerdings auch bei Levofloxacin }\end{array}$ \\
\hline $\begin{array}{l}\text { Toxische Effekte auf Bindegewebsstrukturen } \\
\text { (Tendinopathie, evtl. Arthropathie, } \\
\text { tierexperimentell Chondrotoxizität) }\end{array}$ & $\begin{array}{l}\text { alle, wahrscheinlich } \\
\text { v.a. Ofloxacin und Levofloxacin }\end{array}$ \\
\hline
\end{tabular}

\section{Tabelle 4}

Risikofaktoren für Quinolon-assoziierte Tendinopathien $[11,12]$.

$$
\begin{aligned}
& \text { - gleichzeitiger Gebrauch von Kortikosteroiden; } \\
& \text { - starke mechanische Belastung bei Sportlern, } \\
& \text { wie z. B. Badmintonspielern; } \\
& \text { - Alter > } 60 \text { Jahren; } \\
& \text { - Niereninsuffizienz. }
\end{aligned}
$$

die Unterschiede zur Referenztherapien mutmasslich wegen zu kleiner Umfänge statistisch nicht signifikant (Tab. 6). Betreffend Tendinitiden ausserhalb der Achillessehnen wurde keine klare Assoziation zur Quinolontherapie gefunden.

Über Tendinopathien bei neueren Quinolonen liegen uns derzeit keine publizierten epidemiologischen Untersuchungen vor. Frequenzen von Nebenwirkungsmeldungen zeigen aber eine auffallende Häufung unter Levofloxacin (Tab. 7). Diese Häufung wurde vor allem in der Schweiz, in Frankreich, Belgien, Schweden, England und Irland beobachtet und ist ein Signal für ein möglicherweise speziell erhöhtes Risiko. Levofloxacin ist das hauptsächliche antimikrobiell wirksame Isomer des Racemates Ofloxacin und wird in der Schweiz normalerweise 2,5mal höher (in bezug auf das Racemat) bzw. 5mal höher (in bezug auf das Isomer) als Ofloxacin dosiert, was vermehrte Tendinopathien erklären könnte. Unterschiede zwischen einzelnen Ländern sind wahrscheinlich hauptsächlich durch Unterschiede in der Meldedisziplin und der Verschreibungspraxis bedingt (je nach Land werden einzelne Präparate überwiegend in relativ tiefen kumulativen Dosen bei Harnwegsinfekten oder aber in höheren Dosen bei Infektionen der Luftwege eingesetzt). Spontannebenwirkungsmeldungen sind wegen mutmasslich hoher Dunkelziffer und je nach Präparat verschiedener Sensibilisierung der Ärzteschaft für unerwünschte Wirkungen mit Unsicherheiten behaftet, so dass daraus allein noch keine zuverlässigen Schlüsse betreffend die Inzidenz von Tendinopathien bei neueren Quinolonen gezogen werden können.

\section{Schlussfolgerung für die Praxis}

Quinolon-assoziierte Tendinopathien betreffen vor allem die Achillessehnen und treten in bis zur Hälfte der Fälle beidseitig auf. Sie führen in rund einem Drittel zu Sehnenrupturen und stellen deshalb ernstzunehmende Komplikationen dar. Bei Verschreibung eines Quinolonpräparates sollten deshalb die Patienten über die mögliche Komplikation einer Tendinitis orientiert und angewiesen werden, bei entsprechenden Beschwerden die betroffene(n) Sehne(n) zu schonen und rasch den Arzt zu konsultieren. Wird der Verdacht auf eine Tendinitis ärztlicherseits bestätigt, so sollte die Behandlung mit Quinolonen sofort abgebrochen und eine Ruhigstellung der betroffenen Sehne(n), evtl. mit Anpassung einer Schiene, eingeleitet werden. 
Tabelle 5

Tendinopathien (alle Sehnen) bei antibakteriell behandelten Allgemeinpatienten Antiinfektiva.

\begin{tabular}{|c|c|c|}
\hline & \multicolumn{2}{|c|}{ Anzahl Tendinopathien / Anzahl exponierte Patienten } \\
\hline & Netherl.-IPCI 95-96 [15] & UK-P.E.M. 88-93 [16] \\
\hline Ciprofloxacin & $2 / 456$ & $1 / 11477$ \\
\hline Norfloxacin & $1 / 1030$ & $3 / 11110$ \\
\hline Ofloxacin & $4 / 418$ & $11 / 11103$ \\
\hline Quinolone gepoolt & $7 / 1902$ & $15 / 33690$ \\
\hline Nicht-Quinolone* & $15 / 9406$ & $5 / 22525$ \\
\hline
\end{tabular}

Tabelle 6

Häufigkeit Achillestendinitis: Vergleich Quinolone - Nicht-Quinolon-Antibiotika [15].

\begin{tabular}{|c|c|c|}
\hline & Fälle/Risikoperiode (Tage)* & $\begin{array}{l}\text { relatives Risiko } \\
\text { (95\% Konfidenzintervall) }\end{array}$ \\
\hline Ciprofloxacin & $1 / 20461$ & $2,8^{* *}(0,3-25,2)$ \\
\hline Norfloxacin & $0 / 50981$ & - \\
\hline Ofloxacin & $3 / 18929$ & $10,1^{* *}(2,2-46)$ \\
\hline Quinolone gepoolt & $4 / 90371$ & $3,7^{* *}(0,93-15,1)$ \\
\hline Nicht-Quinolone*** & $4 / 458237$ & 1 \\
\hline $\begin{array}{l}\text { * Periode mit Medil } \\
\text { ** Adjustiert für Alte } \\
\text { *** Amoxicillin, Trime }\end{array}$ & $\begin{array}{l}\text { tenexposition plus ein Monat. } \\
\text { hlecht, Steroidbehandlung und } \\
\text { m, Cotrimoxazol oder Nitrofur }\end{array}$ & Konsultationen. \\
\hline
\end{tabular}

Tabelle 7

Pharmacovigilance: Meldungen von Tendinopathien im Vergleich zu allen gemeldeten unerwünschten Arzneimittelwirkungen (UAW), Stand 17. Dezember 2001.

\begin{tabular}{lcccc} 
Meldungen & \multicolumn{2}{c}{$\begin{array}{l}\text { Schweiz (IKS-Datenbank) } \\
\text { Tendinopathie }\end{array}$} & alle UAW & \multicolumn{2}{l}{$\begin{array}{l}\text { Welt (WHO-Datenbank) } \\
\text { Tendinopathie }\end{array}$} & alle UAW \\
\cline { 3 - 3 } & $8(5 \%)$ & 155 & $649(2,2 \%)$ & 29090 \\
\hline Fleroxacin & $1(1 \%)$ & 91 & $9(1,2 \%)$ & 754 \\
\hline Norfloxacin & $2(6 \%)$ & 34 & $163(2,1 \%)$ & 7536 \\
\hline Ofloxacin & $32(41 \%)$ & 79 & $432(1,8 \%)$ & 23990 \\
\hline Levofloxacin & & & $576(7,8 \%)$ & 7432 \\
\hline Moxifloxacin & & & $18(4,5 \%)$ & 4030
\end{tabular}

Vor allem bei Patienten mit Risikofaktoren für eine Tendinopathie (vgl. Tab. 4) sollte bei Applikation eines Quinolon-Antiinfektivums nach Möglichkeit ein Präparat mit geringer Tendinotoxizität gewählt werden. Nach jetzigem Wissensstand kann diese Toxizität für einzelne Präparate noch nicht zuverlässig abgeschätzt werden. Die zur Verfügung stehenden aktuellen Daten deuten darauf hin, dass die Gefahr für
Tendinopathien von den systemisch wirksamen Quinolonen bei Ciprofloxacin relativ klein, bei Ofloxacin und wahrscheinlich noch ausgeprägter bei Levofloxacin relativ gross ist.

Die Sehnenschädigungen unter Quinolonen wurden auch dank Spontanmeldungen erkannt. Die Fachleute sind aufgerufen, den regionalen Pharmacovigilance-Zentren unerwünschte Wirkungen auf dem neuen gelben Formular (u.a. verfügbar im Anhang des Kompendiums oder auf der Swissmedic-Homepage) zu melden.

Dieser Artikel ist abrufbar auf www.swissmedic.ch.

\section{Literatur}

1 Ball P. Safety of the new fluoroquinolones compared with ciprofloxacin. J Chemother 2000;12 S1:8-11.

2 MedWatch-December 2001: Safety-related drug labeling changes http://www.fda.gov/medwatch/ SAFETY/2001/dec01.htm.

3 Frothingham R. Rates of torsades de pointes associated with ciprofloxacin, ofloxacin, levofloxacin, gatifloxacin, and moxifloxacin. Pharmacotherapy 2001;21:1468-72.

4 Jorgensen C, Anaya JM, Didry C, Canovas F, Serre I, Baldet P et al. Arthropathy with Achilles tendon involvement induced by perfloxacin. Apropos of a case. Rev Rhum Mal Osteoartic 1991;58:623-5.

5 Shakibaei M, Pfister K, Schwabe R, Vormann J et al. Ultrastructure of Achilles tendons of rats treated with ofloxacin and fed a normal or magnesium-deficient diet. Antimicrob Agents Chemother 2000;44:261-6.

6 Stahlmann R, Shakibaei M. Fluorchinoloninduzierte Tendopathien - klinische und experimentelle Aspekte. Chemother J 2000;9:140-7.

7 Williams RJ, Attia E, Wickiewicz TL, Hannafin JA. The effect of ciprofloxacin on tendon, paratendon, and capsular fibroblast metabolism. Am J Sports Med 2000;28:364-9.

8 Kashida Y, Kato M. Characterization of fluoroquinolone-induced Achilles tendon toxicity in rats; comparison of toxicities of 10 fluoroquinolones and effects of anti-inflammatory compounds. Antimicrob Agents Chemother 1997;41:2389-93.

9 Bailey RR, Kirk JA, Peddie BA. Norfloxacin-induced rheumatic disease. N Z Med J 1983;96:590.

10 Perrot S, Ziza JM, De Bourran-Cauet G, Desplaces N, Lachand AT. A new complication related to quinolones: rupture of Achilles tendon. Presse Med 1991;20(26):1234.

11 Royer RJ, Pierfitte C, Netter P. Features of tendon disorders with Fluoraquinolones. Therapie 1994; 49:75-6.

12 Harrell RM. Fluroquinolone-induced tendinopathy: what do we know? South Med J 1999;92:622-5. 
13 Maffulli N, Waterston SW, Squair J, Reaper J, Douglas AS. Changing incidence of Achilles tendon rupture in Scotland: a 15-year study. Clin J Sport Med 1999;9(3):157-60.

14 Houshian S, Tscherning T, Riegels-Nielsen P. The epidemiology of Achilles tendon rupture in a Danish county. Injury 1998;29(9):651-4.
15 van der Linden, van de Lei, Nab HW, Knol A, Stricker BHC. Achilles tendinitis associated with floroquinolones. Br J Clin Pharmacol 1999; 88:433-7.

16 Wilton LV, Pearce GL, Mann RD. A comparison of ciprofloxacin, norfloxacin, ofloxacin, azithromycin and cefixime examined by observational cohort studies. Br J Clin Pharmacol 1996;41:277-84.

\title{
Fluoroquinolones et tendinopathies: un effet de classe?
}

\author{
B. Schnyder, P. Caduff
}

Correspondance:

Dr Benno Schnyder

Swissmedic

Schweizerisches Heilmittelinstitut

Erlachstrasse 8

CH-3000 Bern 9

e-mail:

benno.schnyder@swissmedic.ch

\section{Introduction}

Les fluoroquinolones sont des anti-infectieux relativement bien tolérés. Toutefois, les différents antibactériens appartenant à la famille des quinolones diffèrent considérablement les uns des autres non seulement du point de vue de l'action antibactérienne, mais aussi de la toxicité [1-3]. Tandis que certaines préparations se sont avérées en pratique bien tolérées et sûres, d'autres ont entraîné des effets indésirables, dont certains sont inacceptables (tab. 2). Avant d'opter pour un traitement aux quinolones, il faudrait donc tenir compte du profil spécifique des effets indésirables (tab. 3). Jusqu'à présent, cette question n'a pratiquement pas été traitée dans la littérature en ce qui concerne les tendinopathies.

\section{Aspects pathophysiologiques et précliniques}

Le mécanisme pathologique des tendinopathies associées aux fluoroquinolones n'est pas bien connu. La littérature fait principalement état d'effets toxiques directs, de processus ischémiques [4] et/ou de la chélation par ions de magnésium ou d'autres ions, induisant un manque local de magnésium dans les structures tissulaires mal irriguées telles que tendons ou cartilages, avec des lésions qui en résultent [5,6]. Dans les études précliniques, les fluoroquinolones présentent une toxicité sur le cartilage articulaire immature en relation avec des troubles de la croissance, une inhibition de la synthèse du collagène ou des effets cytotoxiques sur les tendinocytes [7]. Les essais sur animaux ont mis en évidence des différences d'effets sur la structure du tissu conjonctif selon les préparations. Chez les rats, la toxicité tendineuse était la suivante: fléroxacine $=$ péfloxacine $>$ ofloxacine $=$ lévofloxacine $>$ norfloxacine $=$ ciprofloxacine [8]. Etant donné les différences spécifiques considérables au niveau du métabolisme et de l'irrigation sanguine entre l'animal et l'être humain, ces résultats ne peuvent toutefois pas être reportés tels quels à l'être humain.

\section{Aspects cliniques}

La première description d'une tendinite en association avec des quinolones remonte à 1983 [9] et celle d'une rupture tendineuse, à 1991 [10]. Plusieurs facteurs de risque supplémentaires ont été identifiés en ce qui concerne la survenue d'une tendinopathie associée à une quinolone (tab. 4).

Dans un collectif de 100 patients souffrant d'une tendinite d'Achille associée à une quinolone [11], l'intervalle temporel entre le début du traitement et l'apparition des symptômes a varié de 1 à 90 jours, la moyenne étant de 13 jours. L'effet indésirable s'est manifesté essentiellement au niveau du tendon d'Achille. Dans presque la moitié des cas, l'inflammation est ap- 
Tableau 1

Quinolones actuellement commercialisées en Suisse.

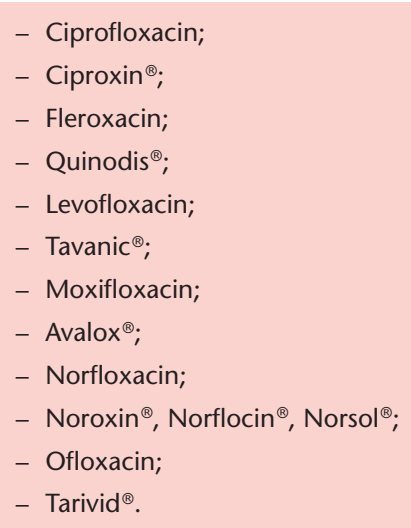

Tableau 2

Quinolones avec effets indésirables inacceptables.

\begin{tabular}{|c|c|c|}
\hline Témafloxacine & $\begin{array}{l}\text { retirée du marché américain en 1992, } \\
\text { jamais commercialisée en } \mathrm{CH}\end{array}$ & $\begin{array}{l}\text { Symptomatique complexe } \\
\text { avec hypoglycémies, } \\
\text { anémies hémolytiques, etc. }\end{array}$ \\
\hline Sparfloxacine & retirée du marché CH en 1997 & Cardiotoxicité et phototoxicité \\
\hline Trovafloxacine & enregistrement radié en 1999 & Hépatotoxicité \\
\hline Grepafloxacine & retirée du marché en 1999 & $\begin{array}{l}\text { Cardiotoxicité et toxicité } \\
\text { gastro-intestinale }\end{array}$ \\
\hline Clinafloxacine & développement stoppé & Phototoxicité \\
\hline
\end{tabular}

Tableau 3

Effets indésirables typiques des quinolones [1-3].

\begin{tabular}{|c|c|}
\hline Intolérance gastrointestinale & toutes \\
\hline Réactions cutanées (phototoxicité, exanthème) & toutes, surtout sous fléroxacine \\
\hline $\begin{array}{l}\text { Réactions au niveau du SNC (maux de tête, } \\
\text { obnubilation, vertige, insomnie, } \\
\text { réactions psychotiques, crampes) }\end{array}$ & $\begin{array}{l}\text { toutes, surtout sous fléroxacine } \\
\text { et ofloxacine }\end{array}$ \\
\hline Toxicité hépatique & toutes \\
\hline Cardiotoxicité avec allongement du QTc & $\begin{array}{l}\text { sous moxifloxacine et récemment } \\
\text { également sous lévofloxacine }\end{array}$ \\
\hline $\begin{array}{l}\text { Effets toxiques sur les structures du tissu conjonctif } \\
\text { (tendinopathie, év. arthropathie, } \\
\text { chondrotoxicité expérimentale chez l'animal) }\end{array}$ & $\begin{array}{l}\text { toutes, vraisemblablement surtout } \\
\text { sous ofloxacine et lévofloxacine }\end{array}$ \\
\hline
\end{tabular}

Tableau 4

Facteurs de risque pour les tendinopathies associées aux quinolones $[11,12]$.

- utilisation simultanée de corticostéroïdes;
- forte charge mécanique chez les sportifs
comme par exemple joueurs de badminton;
- âge > 60 ans;
- insuffisance rénale.

parue des deux côtés. Dans 31\% des cas, il y a eu rupture. Les douleurs sont aiguës et fréquemment caractérisées par des douleurs en cas de mouvement et de palpation. Des douleurs fortes et poignantes indiquent une menace de rupture. Les analyses supplémentaires possibles sont l'ultrasonographie et l'IRM [12].

\section{Epidémiologie}

Telle qu'elle est décrite dans la littérature, l'incidence dans la population des ruptures du tendon d'Achille varie considérablement, selon la période temporelle et l'étude considérées, entre 0,47 et 3,7/10000 [13, 14]. Deux études de cohorte ont porté sur l'incidence des tendinopathies dans une large population de patients sous antibiotiques $[15,16]$. Toutes deux ont révélé une incidence tendanciellement supérieure sous fluoroquinolones ainsi que de fortes différences spécifiques selon les quinolones. L'incidence des ruptures parmi les préparations examinées était maximale sous ofloxacine (tab. 5). Dans l'une de ces études [15], le risque relatif a été calculé par rapport aux traitements de référence (amoxicilline, triméthoprime, cotrimoxazole ou nitrofurantoïne); pour la tendinite d'Achille sous ofloxacine, il se montait à 10,1 (intervalle de confiance à $95 \%: 2,2-46$ ), tandis que, sous ciprofloxacine, norfloxacine et pour les combinaisons des trois quinolones, les différences par rapport au traitement de référence étaient statistiquement non significatives en raison d'échantillons trop petits (tab. 6). En ce qui concerne les tendinites ne concernant pas le tendon d'Achille, aucune association claire avec les quinolones n'a été mise en évidence.

Jusqu'à présent, nous n'avons pas connaissance d'études épidémiologiques concernant les tendinopathies en association avec les nouvelles fluoroquinolones. La fréquence des annonces d'effets spontanées démontre une accumulation frappante sous lévofloxacine surtout en Suisse, en France, en Belgique, en Suède, en Angleterre et en Irlande et signale la possibilité d'un risque élevé (tab. 7). La lévofloxacine est le principal isomère antimicrobien efficace du racémate d'ofloxacine; en Suisse, elle est normalement dosée 2,5 fois plus (par rapport au racémate), voire 5 fois plus (par rapport à l'isomère) que l'ofloxacine, ce qui expliquerait l'accumulation des annonces. Selon toute vraisemblance, les différences d'un pays à l'autre découlent surtout de pratiques différentes en matière d'annonce des 
Tableau 5

Tendinopathies (tous les tendons) chez les patients de médecine générale traités aux antibactériens.

\begin{tabular}{|c|c|c|}
\hline \multicolumn{3}{|c|}{ Nombre de tendinopathies / nombre de patients exposés } \\
\hline & Netherl.-IPCI 95-96 [15] & UK-P.E.M 88-93 [16] \\
\hline Ciprofloxacine & $2 / 456$ & $1 / 11477$ \\
\hline Norfloxacine & $1 / 1030$ & $3 / 11110$ \\
\hline Ofloxacine & $4 / 418$ & $11 / 11103$ \\
\hline Quinolone en pool & $7 / 1902$ & $15 / 33690$ \\
\hline Non-quinolone* & $15 / 9406$ & $5 / 22525$ \\
\hline
\end{tabular}

Tableau 6

Tendinites d'Achille: comparaison antibiotiques avec quinolones - antibiotiques sans quinolones [15].

\begin{tabular}{|c|c|c|}
\hline & Cas/période de risque (jours)* & $\begin{array}{l}\text { Risque relatif } \\
\text { (intervalle de confiance à } 95 \% \text { ) }\end{array}$ \\
\hline Ciprofloxacine & $1 / 20461$ & $2,8^{* *}(0,3-25,2)$ \\
\hline Norfloxacine & $0 / 50981$ & - \\
\hline Ofloxacine & $3 / 18929$ & $10,1^{\star *}(2,2-46)$ \\
\hline Quinolone en pool & $4 / 90371$ & $3,7^{* *}(0,93-15,1)$ \\
\hline Non-quinolone ${ }^{* \star *}$ & $4 / 458484$ & 1 \\
\hline \multicolumn{3}{|c|}{ * Période avec exposition médicamenteuse plus un mois. } \\
\hline \multicolumn{3}{|c|}{ ** Ajusté selon l'âge, le sexe, le traitement aux stéroïdes et le nombre de consultations. } \\
\hline
\end{tabular}

Tableau 7

Pharmacovigilance: annonces de tendinopathies en rapport avec tous les effets indésirables annoncés (EI), état au 17 décembre 2001.

\begin{tabular}{|c|c|c|c|c|}
\hline \multirow[t]{2}{*}{ Annonces } & \multicolumn{2}{|c|}{ Suisse (base de données OICM) } & \multicolumn{2}{|c|}{ Monde (base de données OMS) } \\
\hline & Tendinopathie & Total des El & Tendinopathie & Total des EI \\
\hline Ciprofloxacine & $8(5 \%)$ & 155 & $649(2,2 \%)$ & 29090 \\
\hline Fléroxacine & & & $9(1,2 \%)$ & 754 \\
\hline Norfloxacine & 1 (1\%) & 91 & $163(2,1 \%)$ & 7536 \\
\hline Ofloxacine & $2(6 \%)$ & 34 & $432(1,8 \%)$ & 23990 \\
\hline Lévofloxacine & $32(41 \%)$ & 79 & $576(7,8 \%)$ & 7432 \\
\hline Moxifloxacine & & & $18(4,5 \%)$ & 4030 \\
\hline
\end{tabular}

effets secondaires et en matière de prescription (selon le pays, certaines préparations sont utilisées à des doses cumulatives relativement faibles pour les infections des voies urinaires, mais à des doses supérieures pour les infections des voies respiratoires). En raison d'un taux vraisemblablement élevé de cas non-déclarés et d'une sen- sibilisation différente du corps médical selon les préparations, les annonces d'effets secondaires ne peuvent pas être considérées comme vraiment représentatives, de sorte qu'il n'est pas encore possible d'en tirer des conclusions définitives en ce qui concerne l'incidence des tendinopathies sous quinolones récentes.

\section{Conclusions pour la pratique}

Les tendinopathies associées aux quinolones concernent surtout le tendon d'Achille et, dans la moitié des cas, surviennent des deux côtés. Dans un tiers des cas, elles conduisent à des ruptures du tendon et représentent de ce fait une complication à prendre au sérieux. En cas de prescription d'une préparation aux quinolones, les patients devraient donc être informés d'une possible complication sous la forme de tendinites et avertis du fait qu'il leur faut ménager le(s) tendon(s) concerné(s) en cas de douleurs et consulter un médecin dans les meilleurs délais. Si le médecin confirme la présomption de tendinite, le traitement aux quinolones doit être interrompu sans délai et tout tendon concerné doit être immobilisé, évent. à l'aide d'une gouttière.

Il convient donc de choisir une préparation à faible toxicité tendineuse en cas d'administration d'un antibiotique à base de quinolone chez les patients présentant les facteurs de risque spécifiques de la tendinopathie (cf. tab. 4). En l'état actuel des connaissances, nous ne pouvons pas estimer exactement le risque de tendinopathies dues aux différentes préparations. Les données actuelles indiquent que la toxicité tendineuse en association avec des fluoroquinolones systémiques est relativement faible sous ciprofloxacine, relativement grande sous ofloxacine et vraisemblablement encore plus marquée sous lévofloxacine.

Les tendinopathies en association avec des fluoroquinolones ont été relevées grâce aux annonces d'effets spontanées. Nous invitons les médecins à annoncer les effets secondaires aux centres régionaux de pharmacovigilance au moyen du formulaire jaune (notamment disponible en annexe du Compendium ou sur le site de Swissmedic).

Cet article est disponible à l'adresse www.swissmedic.ch.

Traduction: A. Meyer 


\section{Références}

1 Ball P. Safety of the new fluoroquinolones compared with ciprofloxacin. J Chemother 2000;12 S1:8-11.

2 MedWatch-December 2001: Safety-related drug labeling changes http://www.fda.gov/medwatch/ SAFETY/2001/dec01.htm.

3 Frothingham R. Rates of torsades de pointes associated with ciprofloxacin, ofloxacin, levofloxacin, gatifloxacin, and moxifloxacin. Pharmacotherapy 2001;21:1468-72.

4 Jorgensen C, Anaya JM, Didry C, Canovas F, Serre I, Baldet P et al. Arthropathy with Achilles tendon involvement induced by perfloxacin. Apropos of a case. Rev Rhum Mal Osteoartic 1991;58:623-5.

5 Shakibaei M, Pfister K, Schwabe R, Vormann J et al. Ultrastructure of Achilles tendons of rats treated with ofloxacin and fed a normal or magnesium-deficient diet. Antimicrob Agents Chemother 2000;44:261-6.

6 Stahlmann R, Shakibaei M. Fluorchinoloninduzierte Tendopathien - klinische und experimentelle Aspekte. Chemother J 2000;9:140-7.

7 Williams RJ, Attia E, Wickiewicz TL, Hannafin JA. The effect of ciprofloxacin on tendon, paratendon, and capsular fibroblast metabolism. Am J Sports Med 2000;28:364-9.

8 Kashida Y, Kato M. Characterization of fluoroquinolone-induced Achilles tendon toxicity in rats; comparison of toxicities of 10 fluoroquinolones and effects of anti-inflammatory compounds. Antimicrob Agents Chemother 1997;41:2389-93.
9 Bailey RR, Kirk JA, Peddie BA. Norfloxacin-induced rheumatic disease. N Z Med J 1983;96:590.

10 Perrot S, Ziza JM, De Bourran-Cauet G, Desplaces N, Lachand AT. A new complication related to quinolones: rupture of Achilles tendon. Presse Med 1991;20(26):1234.

11 Royer RJ, Pierfitte C, Netter P. Features of tendon disorders with Fluoraquinolones. Therapie 1994; 49:75-6.

12 Harrell RM. Fluroquinolone-induced tendinopathy: what do we know? South Med J 1999;92:622-5.

13 Maffulli N, Waterston SW, Squair J, Reaper J, Douglas AS. Changing incidence of Achilles tendon rupture in Scotland: a 15-year study. Clin J Sport Med 1999;9(3):157-60.

14 Houshian S, Tscherning T, Riegels-Nielsen P. The epidemiology of Achilles tendon rupture in a Danish county. Injury 1998;29(9):651-4.

15 van der Linden, van de Lei, Nab HW, Knol A, Stricker BHC. Achilles tendinitis associated with floroquinolones. Br J Clin Pharmacol 1999; 88:433-7.

16 Wilton LV, Pearce GL, Mann RD. A comparison of ciprofloxacin, norfloxacin, ofloxacin, azithromycin and cefixime examined by observational cohort studies. Br J Clin Pharmacol 1996;41:277-84. 